\title{
Nectin like-5 overexpression correlates with the malignant phenotype in cutaneous melanoma
}

\author{
Valentina Bevelacqua ${ }^{1}$, Ylenia Bevelacqua ${ }^{2}$, Saverio Candido스 ${ }^{1}$ Evangelia \\ Skarmoutsou ${ }^{1}$, Alfredo Amoroso ${ }^{1}$, Claudio Guarneri ${ }^{3}$, Angela Strazzanti ${ }^{4}$, Pietro \\ Gangemi $^{5}$, Maria C. Mazzarino ${ }^{1}$, Fabio D'Amico ${ }^{1}$, James A. McCubrey ${ }^{6}$, Massimo \\ Libra $^{1, *}$ and Grazia Malaponte ${ }^{1, *}$ \\ ${ }^{1}$ Section of pathology and Oncology, Department of Bio-medical Sciences, University of Catania, Catania, Italy \\ 2 Section of Plastic Surgery, Department of Medicine and Surgery Specialities, University of Catania, Italy \\ ${ }^{3}$ Section of Dermatology, Department of Social Territorial Medicine, University of Messina, Messina, Italy \\ ${ }^{4}$ Department of Surgery, Azienda Ospedaliero-Universitaria Vittorio Emanuele-Ferrarotto-S Bambino, Catania, Italy \\ ${ }^{5}$ Pathology Unit, Azienda Ospedaliero-Universitaria Vittorio Emanuele-Ferrarotto-S Bambino, Catania, Italy \\ ${ }^{6}$ Brody School of Medicine at East Carolina University, Department of Microbiology \& Immunology, Greenville, NC, USA \\ * Co-last author and co-corresponding author
}

Correspondence to: Massimo Libra, email: mlibra@unict.it

Keywords: NECL-5, melanoma, nevus, skin

Received: August 08, 2012, Accepted: August 24, 2012, Published: August 26, 2012

Copyright: $\odot$ Bevelacqua et al. This is an open-access article distributed under the terms of the Creative Commons Attribution License, which permits unrestricted use, distribution, and reproduction in any medium, provided the original author and source are credited.

\section{ABSTRACT:}

NECL-5 is involved in regulating cell-cell junctions, in cooperation with cadherins, integrins and platelet-derived growth factor receptor, that are essential for intercellular communication. Its role in malignant transformation was previously described. It has been reported that transformation of melanocytes is associated with altered expression of adhesion molecules suggesting the potential involment of NECL5 in melanoma development and prognosis. To shed light on this issue, the expression and the role of NECL-5 in melanoma tissues was investigated by bioinformatic and molecular approaches. NECL-5 was up-regulated both at the mRNA and the protein levels in WM35, M14 and A375 cell lines compared with normal melanocytes. A subsequent analysis in primary and metastatic melanoma specimens confirmed "in vitro" findings. NECL-5 overexpression was observed in 53 of 59 (89.8\%) and 12 of $12(100 \%)$, primary melanoma and melanoma metastasis, respectively; while, low expression of NECL-5 was detected in 12 of 20 (60\%) benign nevi. A significant correlation of NECL-5 overexpression was observed with most of known negative melanoma prognostic factors, including lymph-node involvement $(P=0.009)$ and thickness $(P=\mathbf{0 . 0 0 4})$. Intriguingly, by analyzing the large series of melanoma samples in the Xu dataset, we identified the transcription factor YY1 among genes positively correlated with NECL-5 $(r=0.5)$. The concordant computational and experimental data of the present study indicate that the extent of NECL-5 expression correlates with melanoma progression.

\section{INTRODUCTION}

Cutaneous melanoma represents the most aggressive and lethal malignancy of the skin. Despite advances in melanoma treatment [1-4], mortality from melanoma is still increasing [5]. Melanoma prevention and early detection represent the best approaches to survive [6]. Discovery of biomarkers and their application, in conjunction with traditional cancer diagnosis, staging, and prognosis, could improve early diagnosis and patient care [7-9]. Thus, there is an urgent need to develop prognostic biomarkers that can differentiate between malignant and non-malignant skin lesions and identify melanoma 
patients with high-risk primary lesions to facilitate greater surveillance.

Many pathways were identified to play a role in melanoma development [10-11]. It is now well known that up- or downregulation of several adhesion molecules, such as N- and E-cadherin, MCAM (melanoma cell adhesion molecule), VCAM (vascular cell adhesion molecule), integrins, can be involved in the progression of several cancer types [12-16], including melanoma [17-19]. In this context, emphasis has recently been placed on the cell adhesion molecule, nectin like molecule-5 (NECL5), also named NECL-5/NECL-5/CD155/ Tage4 [20-23]. Experimental studies demonstrated that up-regulated NECL-5 enhances cell movement and proliferation and that cell-cell contacts do not induce a reduction of NECL5 at the cell surface of NIH3T3 transformed cells (mouse embryonic fibroblast cell line) by oncogenic Ki-Ras; it was also suggested that up-regulation of NECL-5, mediated by activator protein-1 (AP1) pathway, might be involved in the loss of contact inhibition in transformed cells [2428]. Previous data showed that the down-regulation of NECL-5 in cancer cells decreases migration, proliferation and metastasis $[25,29]$. In the mean time, its upregulation has been observed in cancer cell lines [28-30], in human tumors such as glioblastoma, ovarian carcinoma, prostate, colorectal and lung cancer [31-35]. However, the

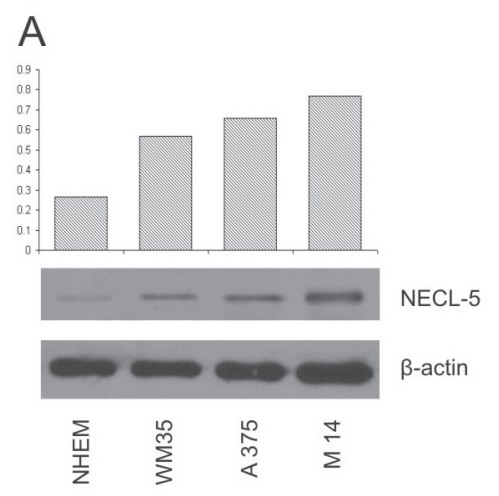

C
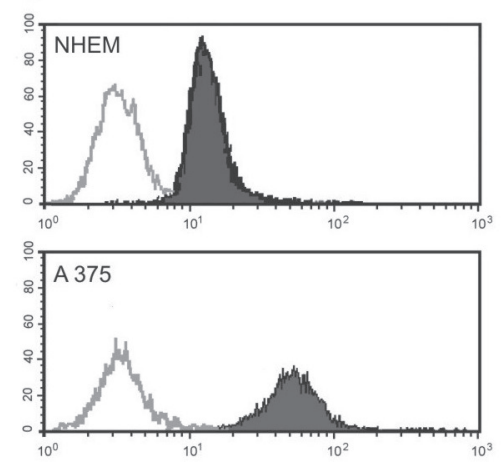

involvement of NECL-5 in human cutaneous melanoma is still lacking. Overall, these data led us to investigate if NECL-5 expression may play a role in melanoma development and progression.

In the present study, NECL-5 expression was observed at different levels in tissue samples from normal skin, benign melanocytic nevi and primary and metastatic melanoma suggesting that NECL-5 may be a potential marker of melanoma progression.

\section{RESULTS}

\section{Computational analysis of NECL-5 gene in melanoma datasets}

Transcript levels were higher in primary and in metastatic melanoma samples when compared to those of normal samples by analyzing several public available melanoma datasets (Supplementary Figure 1).

\section{NECL-5 is overexpressed in melanoma cell lines}

We first analyzed the NECL-5 expression by western blot in WM35, M14 and A375 cell lines, compared with
B
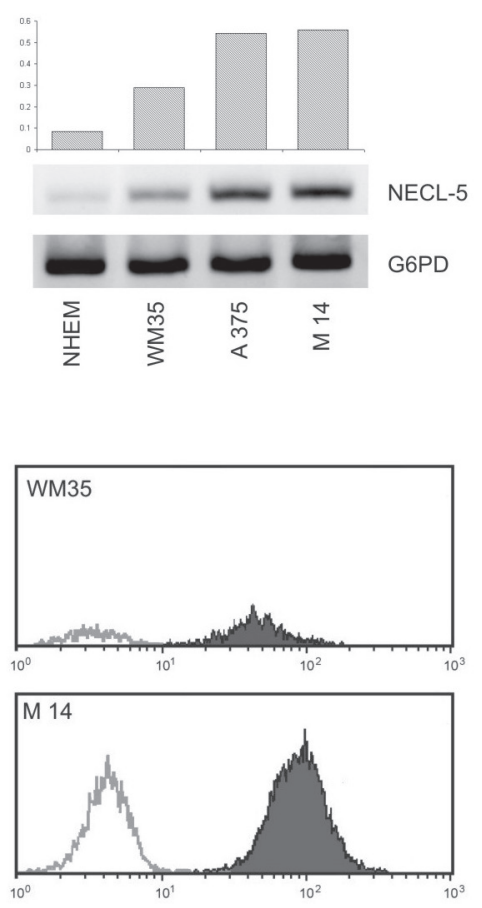

Figure 1: Expression analyses of NECL-5 in WM35, M14 and A375 cell lines. 1A) Western blot in WM35, M14 and A375 cell lines, compared with NHEM. The upper bands represent NECL-5 protein, and the lower bands are $\beta$-Actin used as an internal control. The intensities of these bands were quantified by the Kodak 1D image analysis software. 1B) mRNA expression of NECL-5 gene by RT-PCR using G6PD for normalization. 1C) Flow cytometry analysis of NECL-5 expression. Histograms compare staining with specific antibody (black line) with the appropriate isotype control antibody (gray line). 
NHEM. NECL-5/ $\beta$-Actin ratios were calculated for each of the three experiments and the average was used for the graph (Figure 1A). Although, western blots showed that NECL-5 is expressed in all of the cell lines, its expression in WM35 cells was significantly higher than that in NHEM cells $(p<0.001)$. In addition, analysis with oneway ANOVA showed that NECL-5 protein levels were significantly higher in M14 and A375 cells compared to those in WM35 cells $(p=0.002)$. As expected, NECL-5 mRNA levels were higher in M14 and A375 cells than in both WM35 and NHEM cells (Figure 1B). These results were also confirmed by flow cytometry analysis (Figure 1C). Finally, IHC evaluation showed that NECL-5 protein is expressed in all melanoma cell lines (Supplementary Figure 2). In detail, NECL-5 immunolabelling was mainly localized at both extracellular and intracellular level of WM35, A375 and M14 cells and a stronger immunolabelling was observed in metastatic cell lines (Supplementary Table 2).

\section{siRNA of NECL-5 results in decreased melamoma cell migration}

Knockdown of NECL-5 gene in both A375 and M14 cells was used to understand the effect on cell migration. As shown in Figure 2, the transfection of NECL-5-specific siRNAs cause an almost $70 \%$ loss of NECL-5 transcript levels compared to control both in A375 and M14 cells. A reduction of $40 \%$ of invasive capacity was observed when NECL-5 was knocked-down in melanoma cells compared with control cells $(\mathrm{p}<0.001)$.

\section{NECL-5 is overexpressed in melanoma tissues}

IHC evaluation of NECL-5 was also performed in 20 benign nevi and 71 melanoma specimens, including 12 metastatic melanoma (Figure 3). Normal skin tissues showed no immunostaining (Figure 3a) whereas a positive immunostaining was in CMMs specimens (Figure 3c and 3d). The immunopositivity for NECL-5 was also significantly different between the benign (nevi) (Figure $3 \mathrm{~b}$ ) and malignant lesions (primary and metastatic melanomas) (Figure 3c and 3d). Ten of $20(50 \%)$ benign nevi samples had minimal weak immunostaining of NECL-5. It was mainly detected in the outer zones of the rows of melanocytes and, almost absent, in cell-cell contacts between melanocytes (Figure 3b). In 3 nevi, NECL-5 immunostaining was present in the epidermis and upper dermis. Almost $91.5 \%$ of all melanoma specimens (primary and metastatic melanomas) showed an enhanced NECL-5 immunoreactivity (Figure 3c and 3d) when compared with the benign nevi tissue samples (Figure 3b) $(\mathrm{P}<0.0001)$. In primary melanoma, NECL-5 staining was detected in cytoplasm and/or membrane of cancer cells. Immunoreactivity of NECL-5 was observed to be higher, within tumor cells, in metastatic melanomas than in primary melanoma $(\mathrm{P}<0.001)$. A strongly labelled for NECL-5 there were in the papillary dermis, and variably in the reticular dermis and around blood vessels (Figure 3d). As shown in Figure 3e and 3f, NECL-5 expression is higher in melanoma sections with thickness $>1 \mathrm{~mm}$ in comparison with those with thickness $\leq 1 \mathrm{~mm}$.

The immunoreactivity was represented by the assignment of staining index (SI), in which the intensity of immunostaining was multiplied by the extent of positive area in the tissue samples. The mean $\pm \mathrm{SE}$ of the staining index for benign nevi was $2.3 \pm 0.51$; while, it was $7.95 \pm$ 0.45 and $12.6 \pm 1.58$ for primary and metastatic melanoma samples, respectively (Suppl. Figure 3). In this analysis, primary and metastatic melanoma showed significantly higher level of NECL-5 expression than nevi $(\mathrm{P}<0.0001$ for both). As shown in the Table 2, 8 of 20 (40\%) benign nevi were negative, only 2 of $20(10 \%)$ displayed moderate and 10 of $20(50 \%)$ had weak NECL-5 staining. We found that $51 \%$ of primary melanomas samples showed strong (30 of 59 cases) or $27 \%$ moderate ( 16 of 59 ) or $12 \%$ weak (7 of 59) immunostaining, whereas 6 of 59 (10\%) were negative and those negative samples were all melanoma in situ. NECL-5 was immunohistochemically detected in all melanoma metastases. Of 12 metastasis, 8 of 12 (67\%) showed strong staining, 3 of $12(25 \%)$ moderate and 1 of $12(8 \%)$ had weak staining intensity.
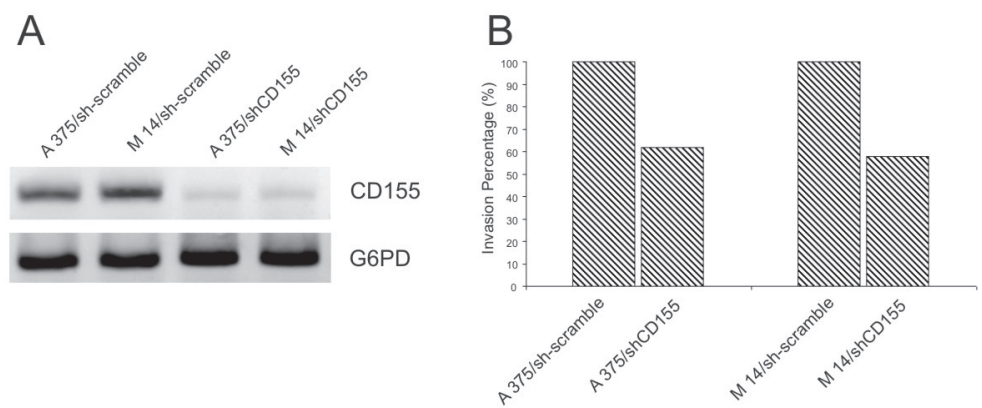

Figure 2: (A) siRNA of NECL-5 gene in both A375 and M14 cells was used to understand the effect on cell migration. (B) siRNA of NECL-5 gene resulted in a significant decreased in transwell migration compared to control cells. 
Table 1: Socio-demographic and clinical characteristics of patients with melanoma and benign nevi.

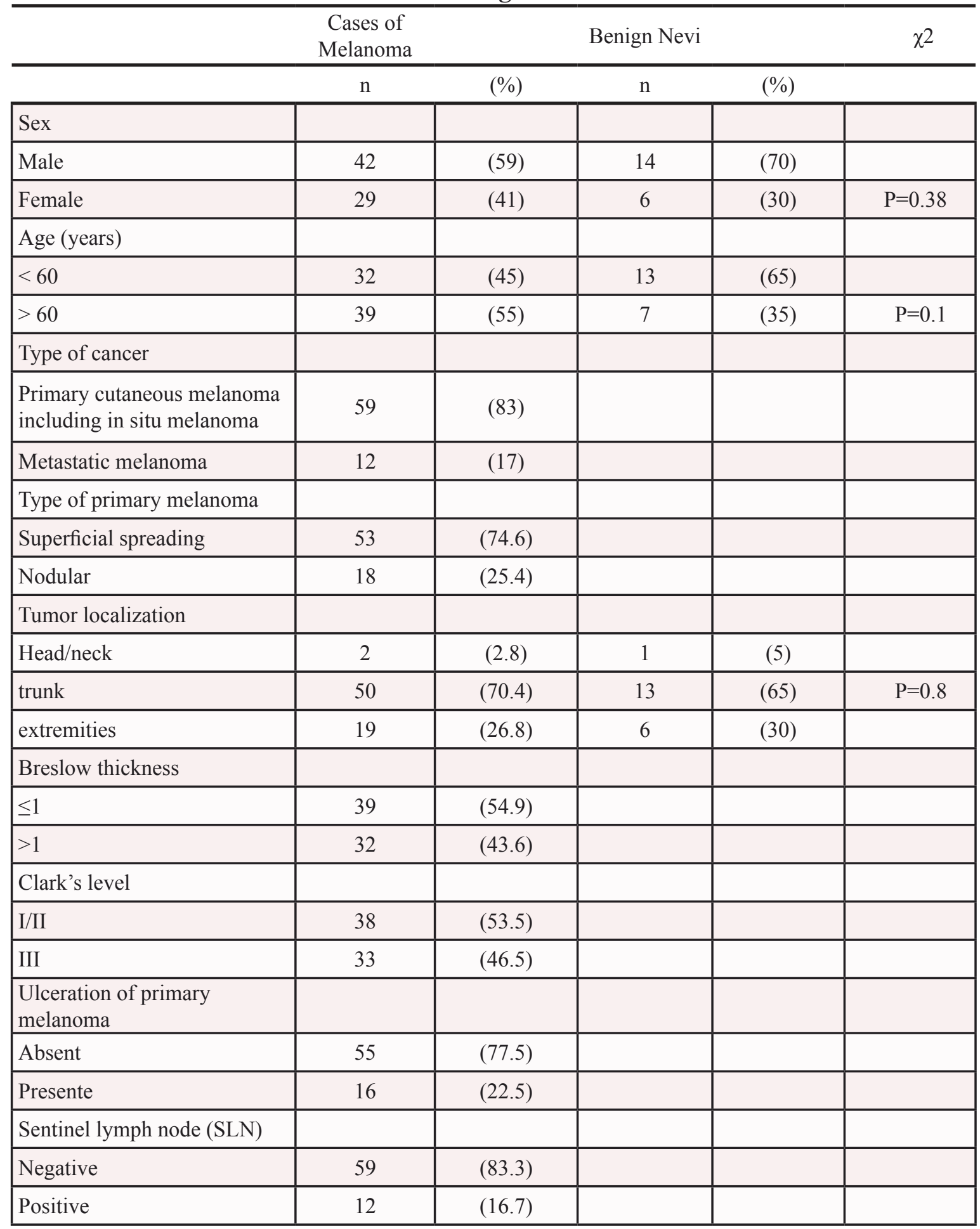

\section{Correlation of NECL-5 expression with prognostic features in melanoma}

We further examined a possible correlations between NECL-5 expression profiles in melanoma samples with their clinicopathologic features. Table 3 shows the potential association of NECL-5 expression levels, stratified according to the median NECL-5 immunoscore $(<9$ and $\geq 9)$ with different clinicopathological features. As shown in Table 3, our analysis in melanoma tissue sections revealed that NECL-5 expression was strongly correlated with lympho node involvement $(\mathrm{P}=0.009)$ and 
Breslow thickness $(\mathrm{P}=0.004)$. Lymph node spreading represents the most common way of metastasis in CMMs. As expected, the comparison between the group of melanoma sections with thickness $>1 \mathrm{~mm}$ and those with thickness $\leq 1 \mathrm{~mm}$ evidenced that NECL-5 immunoreactive scores were higher in thick (score: mean 10.53 $\pm 0.74, \mathrm{P}=$ 0.003 ) than in thin melanomas (score: mean $7.51 \pm 0.59$ ) (Supplemnetary Figure 4a). In particular, 81.2\% (26 of 32) thick melanoma displayed $4+$ of staining, $9.4 \%$ (3 of 32 ) had $3+$ and $9.4 \%$ (3 of 32 ) showed $2+$, while, $28.2 \%$ (11 of 39) thin melanoma had $4+, 25.6 \%$ (10 of 39) had $3+$ and $46.1 \%$ (18 of 39 ) showed $2+$ of NECL-5 staining. NECL-5 immunoscore was directly correlated with Breslow thickness $(r=0.59, \mathrm{P}=0.0001$; Supplemnetary Figures $4 \mathrm{~b}$ and $4 \mathrm{c}$ ). These observations suggested a correlation between increased NECL-5 expression and clinical progression in melanoma. However, no evident

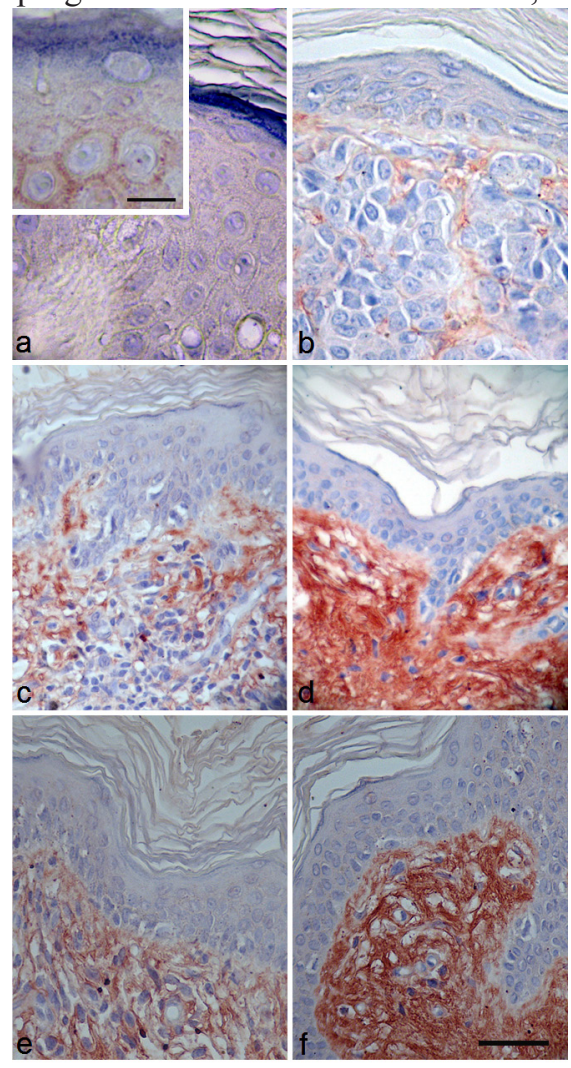

Figure 3: Immunostaining using a specific antiNECL-5 antibody in a representative fraction of human melanocytic lesions of benign nevi, primary and metastatic melanoma. Immunohistochemical analysis of NECL-5 in normal skin, benign nevi and melanoma tissues. a: Normal skin tissue shows no immunostaining for NECL-5 (bar: $35 \mu \mathrm{m}$; inset, $15 \mu \mathrm{m}$ ). b: Skin dermis of benign nevus tissue is weakly labelled for NECL-5 (bar: $32 \mu \mathrm{m}$ ). c: In primary melanoma lesion, immunolabelled melanocytes are immersed in a matrix also immunostained for NECL-5 (bar: $50 \mu \mathrm{m}$ ). d: Dermis and melanocytes of metastatic melanoma skin tissue are strongly labelled for NECL-5 molecule (bar: $50 \mu \mathrm{m}$ ). e: thickness $\leq 1 \mathrm{~mm}$; f: thickness $>1 \mathrm{~mm}$; NECL-5 expression is higher in melanoma sections with thickness $>1 \mathrm{~mm}$ in comparison with those with thickness $\leq 1 \mathrm{~mm}$ (bar: $50 \mu \mathrm{m}$ ). correlations were observed between NECL-5 expression profiles and other clinicopathologic features, including age, sex, type of primary melanoma (superficial-spreading melanoma and nodular melanoma), Clark's levels and ulcerations.

\section{Positive correlation of NECL-5 with YY1 in melanoma}

To further confirm the association of NECL-5 with a more malignant phenotype in melanoma development, a positive correlation between NECL-5 and YY1 transcript levels has been identified by analyzing Oncomine software $(\mathrm{r}=0.468)$ (Figure $4 \mathrm{a})$. In agreement with computational identification, immunohistochemistry evaluation reveals that both NECL-5 and YY1 were concomitantly overexpressed in melanoma samples (Figure 4b).

\section{DISCUSSION}

Cutaneous melanoma is the example of tumor type which progresses through various stages, from benign nevi to metastatic cancer, culminating in an highly aggressive disseminating tumor [40].

To our knowledge, this is the first report showing the expression of NECL-5 in primary and metastatic melanoma tissues and its correlation with some clinicopathologic features by using several independent approaches. These findings suggest its role in melanoma progression. A differential expression of NECL-5 was detected between primary melanoma tissues and benign nevi. Only $60 \%$ of nevi displayed minimal or weak NECL5 expression, whereas $91.5 \%$ of melanoma specimens showed a high NECL-5 expression. The immunopositivity for NECL-5 protein was significantly different between the benign (nevi) and malignant (in situ, invasive and metastatic melanomas) lesions; whereas it was absent in normal skin. In benign nevi NECL-5 expression was mainly present in the outer zones of the rows of melanocytes and seemed to be almost absent in cellcell contacts between melanocytes, even if in three nevi NECL-5 immunostaining was present in the epidermis and upper dermis and declining with increasing depth. It might speculate that NECL-5 expression in nevus cells enables them to invade into the dermis. Benign and atypical nevi have been shown to exist in clinical and histologic contiguity with cutaneous melanoma suggesting that these melanocytic nevi are also susceptible to malignant transformation [41]. These findings are consistent with other published reports describing the hypotetical role of NECL-5 overexpression in different tumors [29,3135]. Most recently, Nakai et al. reported that NECL-5 expression is up-regulated in lung adenocarcinoma and has a negative effect on the prognosis of patients [35]. Previously, Ochiai et al. had shown that CD155 expression 
Table 2: The staining intensity in melanocitic lesion

\begin{tabular}{l|l|l|l|l|}
\hline Melanocytic lesions & \multicolumn{1}{l}{ Immunoscore } & Moderate & \multicolumn{2}{l}{ Weak } \\
\hline Benign nevi & - & $2 / 20(10 \%)$ & $10 / 20(50 \%)$ & $8 / 20(40 \%)$ \\
\hline Primary melanoma & $30 / 59(51 \%)$ & $16 / 59(27 \%)$ & $7 / 59(12 \%)$ & $6 / 59(10 \%)$ \\
\hline Metastatic melanoma & $8 / 12(67 \%)$ & $3 / 12(25 \%)$ & $1 / 12(8 \%)$ & - \\
\hline
\end{tabular}

was up-regulated in several primary breast tumors [30]. Similarly, Sloan et al. observed that elevated espression of CD155 was detected in several primary cancer types [29]. In the current study, knockdown of the gene encoding NECL-5 in A375 and M14 cells caused a reduction of nearly $40 \%$ in tumor invasion capacity and provided further data that underline the role of NECL-5 in the progression of malignant melanoma.

Our analysis in melanoma tissue speciments showed that the increase in NECL-5 immunoreactivity correlated with lymph node involvement and Breslow thickness that are considered to be major predictive factors in melanoma microstaging $[45,46]$. These results revealed that increased NECL-5 expression correlated with more aggressive behaviour. In fact, high NECL-5 immunostaining in the nuclei and cytoplasm of cancer cells was detected in approximately more then $50 \%$ and $83 \%$ of primary melanomas and metastatic samples, respectively. In agreement with our findings, previous study showed that transformed cells gain metastatic ability owing to upregulation of NECL-5 [26,28]. Many studies have demonstrated that overexpression of NECL-5 is implicated in the loosening of contact inhibition and in the loss of epithelial integrity, so this enhances cell proliferation and migration may contribute to the dissemination of cells from tumors [28]. Other substrates of NECL-5 have been associated with cancer development and tumor progression. The loss of E-cadherin in melanocytic lesions would be found initially in nevi and more frequently in melanomas, this process appears to be one of the critical steps in the progression of melanoma [47]. The loss of E-cadherin in melanoma cells could trigger the release of cancer cells from the primary tumor by subsequent breakdown in the melanocytes-keratinocytes interaction and invasion of melanoma cells [47]. This suggests that increased expression of NECL-5 may lead to elevated shedding of E-cadherin and loss of cell-cell contacts. E-cadherinmediated cell adhesion might be abrogated through degradation of the extracellular portion by gelatinases, as matrix metalloproteinases (MMPs)-2 [48]. Interestingly, it has been showed a novel link between NECL-5 and MMP-2 expression in glioblastoma [49]. This study suggested that NECL-5 enhance PI3K/Akt signalling at the leading edge during migration. The signaling guide to MMP-2 production, which can facilitate invasion. MMP-2 is strongly expressed in malignant melanomas and it correlates with invasion and metastatic behavior [50]. Thus, up-regulation of NECL-5 expression may be a common pathway through which cancer cells can acquire a more invasive or dispersive phenotype, a requirement for cancer progression [51].

Furthermore, the implication of NECL-5 with the aggressiveness of melanoma may be corroborate by its correlation with the transcription factor YY1. Our recent data showed that transcript levels of the transcription factor YY1 are higher in several cancer types including melanoma when compared with those of normal tissue [52-55]. We have also observed that the inhibition of YY1 in A375 melanoma cells induces p53-mediated apoptosis after treatment with nitric-oxide donor [56]. These observations have lead us to investigate if YY1 transcript levels correlate with NECL-5 mRNA levels in melanoma. As aspected, by analyzing the large series of melanoma samples in the $\mathrm{Xu}$ dataset, we observed that YY1 is positively correlated with NECL-5 $(r=0.5)$ underlying that its overexpression is associated with a malignant phenotype in melanoma development.

In conclusion, we have provided evidence that NECL-5 may be an important biomarker in the early diagnosis of melanoma, being slightly expressed already in benign nevi. Moreover, NECL- 5 seems to be an indicator of tumour progression in melanoma. NECL-5 is strongly expressed in metastatic melanoma cell lines. In primary melanoma lesions was its expression was localized in the deeper part of the tumors and was consistent with a possible role in the progression toward an invasive phenotype. Limitations of our study is the absence of clinical follow-up, including survival data. Additional studies of the spectrum of expression of NECL-5 and its relationship with other adhesion molecules and growth factors will be necessary to address further the complex processes of pathogenesis and progression in malignant melanoma. Our future goals will be to determine the molecular and cellular consequences as the result of an increased NECL-5 expression. These studies will allow the linking of NECL-5 to specific signalling pathways so that rational mechanism-based therapeutic strategies directed at specific molecular targets can be developed toward the potentially better diagnosis and management 
of melanoma.

\section{MATERIALS AND METHODS}

\section{Gene expression data sets}

Computational evaluation of NECL-5 has been performed by ONCOMINE software. Differential mRNA expression analyses among normal skin tissue versus benign melanocytic skin nevus (BMN), BMN versus cutaneous malignant melanoma (CMM) and CMM versus metastatic melanoma were explored in 3 datasets showing statistical significance less than $0.05(\mathrm{p}<0.05)$ [36-38]. Statistical analysis was accomplished through use of ONCOMINE algorithms. Haqq Melanoma Dataset was excluded from the study as a different microarray platform has been used by the authors [39]. Fold change values were considered for this analysis. Further details

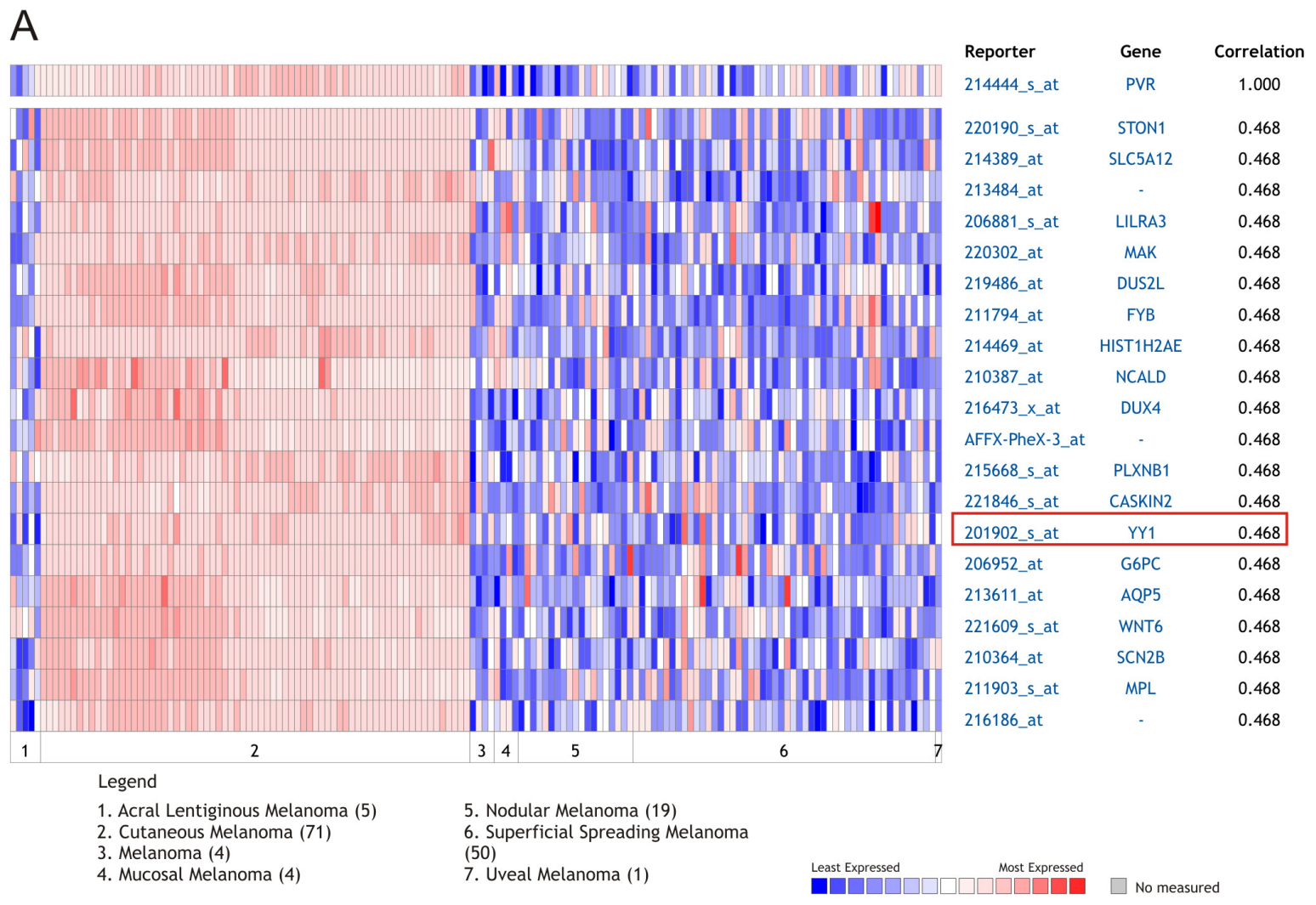

B
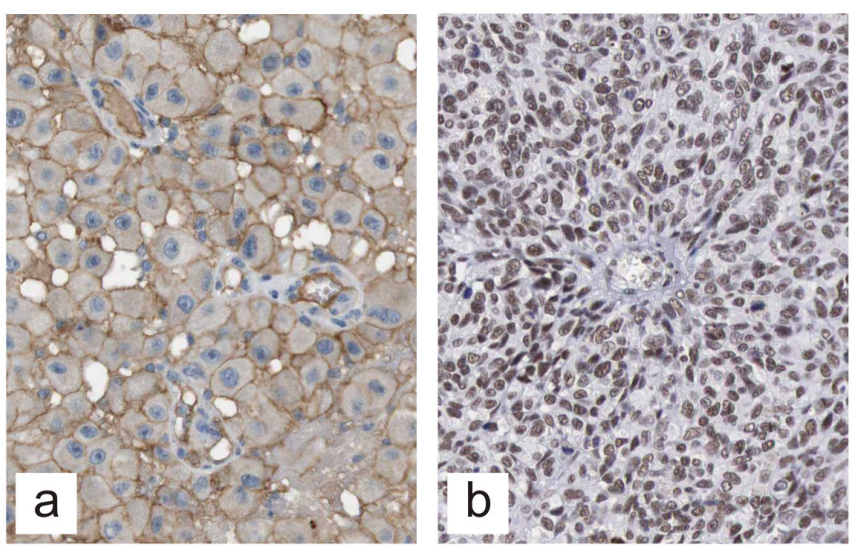

Figure 4: Correlation of YY1 with NECL-5 in melanoma. Heat map of genes positively correlated with NECL-5 $(r=0.5)$ by Pearson correlation analysis in the Xu melanoma dataset (A). Immunohistochemistry evaluation of NECL-5 and YY1 in a representative case of melanoma respectively in Panel (a) and (b) (B). 
on datasets are reported in Supplementary Table 1. Correlation between NECL-5 and Yin Yang 1 (YY1) transcript levels was analyzed according to Oncomine software in the Xu Melanoma Dataset [38].

\section{Cell lines and culture conditions}

Melanoma cell lines (WM35, A375, M14) and human epidermal melanocyte (NHEM) cell line were kindly provided by Dr.V. Russo (Tumor Targeting Research Unit, San Raffaele Scientific Institute, Milano, Italy) and Dr. R. De Maria (Department of Hematology, Oncology and Molecular Medicine, Istituto Superiore di Sanità, Rome, Italy), respectively. Melanocytes and melanoma cell lines were maintained in Melanocyte Growth Medium (Lonza, Walkersville, USA) and RPMI medium (Gibco, Life Technologies Inc., Milan, Italy), supplemented with $2 \mathrm{mmol} / \mathrm{l}$ L-glutamine, 100 IU penicillin and $100 \mu \mathrm{g} / \mathrm{ml}$ streptomycin and $2 \%$ heatinactivated fetal calf serum (Gibco Life Technologies Inc.), respectively. All cell lines were cultured at $37^{\circ} \mathrm{C}$ in $5 \% \mathrm{CO}_{2}$ atmosphere at constant humidity and passaged twice a week. Melanocytes and melanoma cells were grown to subconfluency, harvested and subjected to westen blot, real-time polymerase chain reaction (RT-PCR) or flow cytometry and immunoistochemistry analysis (IHC).

\section{Tissue samples and patients characteristics}

Archival paraffin tissue section of 91 melanocytic lesions excised between 2006 and 2010 were retrieved from the Department of Pathology at the Vittorio Emanuele Hospital, Catania, Italy. The collection and storage of samples were performed according to local ethical guidelines. Institutional review board approval was obtained for this study. There were (i) 59 CMM, including 10 melanoma in situ, (ii) 12 metastatic malignant melanoma, randomly chosen for the study, $(8$ subcutaneous melanoma lesions and 4 regional lymph node metastases), (iii) $20 \mathrm{BMN}$, (iv) 10 normal skin adjacent to different tumour masses. Clinical staging of CMM was performed on a pathological basis according to the new American Joint Committee on Cancer 2001 classification system. Diagnosis and staging of BMN had been performed on the basis of clinical, histopathologic findings. All sections of CMM were superficial spreading melanoma (SSM) and nodular melanoma (NM), at different stages (Clark's level I to III). Localization of the primary melanoma were grouped in three classes: head/neck, trunk, extremities. We stratified CMM by the Breslow tumor thickness as thin $(\leq 1 \mathrm{~mm})$ and thick $(>1 \mathrm{~mm})$. Specimens were fixed in neutral $4 \%$ buffered formaldehyde for a minimum of $24 \mathrm{~h}$ and subsequently embedded in paraffin. The clinicopathological features of 91 melanocytic lesions are summarized in Table 1.

\section{RT-PCR analysis}

Total cellular RNA was extracted from cultured cell lines with Micro-to-Midi total RNA purification system (Invitrogen, Milano, Italy) according to the manufacture's instructions. Reverse transcription was carried out using M-MLV reverse transcriptase (Invitrogen) and random primers (Invitrogen). Semiquantitive PCR was carried out by applying standard condition. The following primers were used $\left(5^{\prime} \rightarrow 3^{\prime}\right)$ : CD155 sense: TATCTGGCTCCGAGTGCTTGCC; CD155 antisense ACGACGGCTGCAAAAGTGGCG; glucose-6-phosphate dehydrogenase (G6PD) sense: ACGTGATGCAGAACCACCTACTG; G6PD antisense: ACGACGGCTGCAAAAGTGGCG. For quantitation, gels were scanned, and the pixel intensity for each band was determined using the Image J program and normalized to the amount of G6PD.

\section{Immunoblot Analysis}

Immunoblot analysis was performed as described by Merrill et al.[31]. Antibodies included anti-CD155 (ab 103630, Abcam, Cambridge, UK) and anti- $\beta$ Actin (Sigma-Aldrich). CD155 antibody binding was detected with streptavidin-horseradish peroxidase complex (Roche, Indianapolis, IN) and visualized using enhanced chemiluminescence substrate (Amersham Life Science, Piscataway, NJ, USA). $\beta$ Actin was used as an internal loading control.

\section{CD155 siRNA}

A double stranded siRNA oligonucleotide targeting CD155 (5'-CAACUUUAAUCUGCAACGUdTdT-3') was chemically synthesized (Dharmacon Research) and transfected into WM35, A375 and M14 cells using Oligofectamine (Invitrogen) following manufacturers instructions using $200 \mathrm{nM}$ siRNA per $10 \mathrm{~cm}$ dish. Cells were incubated with siRNA in OptiMEM (Invitrogen) for 6 hrs after which time normal growth media was added. Cells were then incubated for $72 \mathrm{~h}$ to achieve $>80 \%$ knockdown of CD155. Control cells were transfected with a scrambled siRNA oligonucleotide at matching concentration. After transfection, cells were harvested and subjected to the invasion assay.

\section{Tumor invasion assay}

The in vitro invasion assay was done by using a cell invasion assay kit based on the manufacturer's protocol (Chemicon, Billerica, MA). Control cells and siRNAtreated cells were trypsinized and used for the invasion assay. 


\section{Immunohistochemistry}

Immunohistochemical staining of NECL-5 were done according to standard protocols using CD155 antibody (ab60115, Abcam, Cambridge, UK). Detection of primary antibody was performed using the streptavidinbiotin-peroxidise complex system (Santa Cruz Biotechnology Inc., Santa Cruz, CA)., according to the manufacturer's instructions. The detection was carried out using Histostain-Plus Kit (Zymed, South San Francisco, CA). AEC (3-amino-9-ethylcarbozole) was used as a chromogen. Negative control experiments were performed as above described. Detection of YY1 was performed by using Sigma Aldrich HPA001119 antibody according to the manufacturer's instructions.

\section{Image analysis}

Immunolabelled and sampled tumor sections were observed using a Leica DMRB microscope (10× and 40× magnification) (Leica, Wetzlar, Germany), the images were photographed with a Canon G-9 camera (Canon, Japan) and analyzed using Image $\mathrm{J}$ software. Four randomly chosen fields of view were assessed in the melanoma biopsies and in the cell lines. A section was considered negative or positive according to the absence or presence of cytoplasmic staining. Immunoreactivity was assessed by the amount of positive malignant cells in the area. We used a staining index (SI; values 0-16) with the following formula: $\mathrm{SI}=$ immunostaining intensity $\mathrm{x}$ positive area, where intensities were scored semiquantitatively as follows: $4+$, very strong; $3+$, strong; $2+$, moderate; $1+$, weak and 0 , negative. Positive areas were defined as the epithelial area that showed positive malignant cells for NECL-5 immunoreactivity and was graded on an arbitrary scale as 0 , absent; $1+$, less than $25 \% ; 2+, 25 \%$ to $50 \%$; $3+$, $51 \%$ to $75 \%$; and $4+$, more than $75 \%$. The distribution of staining was classified as focal or diffuse. Localization of staining (membranous or cytoplasmic) was also recorded. Scoring was based upon the consensus of three anatomic pathologists.

\section{Flow cytometry analysis}

Cell lines were washed in PBS. Cell suspension were stained with phycoerythrin conjugated mouse $\mathrm{IgG} 1$ isotype control and mouse IgG1 anti-CD155 (Bioscience, USA). Cells were analyzed using a FACScalibur flow cytometer (Becton Dickinson, USA). After gating cells on a forward scatter/side scatter dot plot window on linear scale, the fluorescence intensity of PE-conjugated isotype control and anti-CD155 labelled cells were analyzed in histograms on FL2 channel with logarithmic scales.

\section{Statistical analysis}

The statistical analyses were performed using SPSS for Windows. Comparison of socio-demographic and clinical characteristics benign nevi and melanoma patients was performed with the $\chi^{2}$ test. Normally distributed data were expressed as media \pm SE and non normally distributed data were expressed as median (range). To correlate NECL-5 staining index and clinicopathologic features was applied the $\chi^{2}$ square test. Additionally, the correlations between NECL-5 expression in melanoma sections and Breslow thickness were assessed by Spearman rank correlation. For semiquantitative RT-PCR analysis, statistics was performed by using the unpaired Student's $t$ test. The statistical differences of NECL5 expression level between primary and metastatic melanoma samples were calculated by Student $t$ test or one-way analysis of variance (ANOVA). Differences were considered to be statistically significant at a level of $\mathrm{p}<$ 0.05 .

\section{GRANT SUPPORT}

ML has been in part supported by a grant from the Italian Ministry of Health, Ricerca Finalizzata Stemness 2008 and by the Italian League Against Cancer.

\section{CONFLICTS OF INTEREST}

The authors declare no conflict of interest.

\section{REFERENCE}

1. Chapman PB, Hauschild A, Robert C, Haanen JB, Ascierto P, Larkin J, Dummer R, Garbe C, Testori A, Maio M, Hogg D, Lorigan P, Lebbe C, Jouary T, Schadendorf D, Ribas A, O’Day SJ, Sosman JA, Kirkwood JM, Eggermont AM, Dreno B, Nolop K, Li J, Nelson B, Hou J, Lee RJ, Flaherty KT, McArthur GA; BRIM-3 Study Group. Improved survival with vemurafenib in melanoma with BRAF V600E mutation. N Engl J Med. 2011; 364:2507-16.

2. Sosman JA, Kim KB, Schuchter L, Gonzalez R, Pavlick AC, Weber JS, McArthur GA, Hutson TE, Moschos SJ, Flaherty KT, Hersey P, Kefford R, Lawrence D, Puzanov I, Lewis KD, Amaravadi RK, Chmielowski B, Lawrence HJ, Shyr Y, Ye F, Li J, Nolop KB, Lee RJ, Joe AK, Ribas A. Survival in BRAF V600-mutant advanced melanoma treated with vemurafenib. N Engl J Med. 2012; 366:707-14

3. Byrne KT, Turk MJ. New perspectives on the role of vitiligo in immune responses to melanoma. Oncotarget. 2011; 2:684-94.

4. Villares GJ, Zigler M, Bar-Eli M. The emerging role of the thrombin receptor (PAR-1) in melanoma metastasis--a possible therapeutic target. Oncotarget. 2011; 2:8-17. 
5. Demierre MF, Sabel MS, Margolin KA, Daud AI and Sondak VK. State of the science 60th anniversary review: 60 years of advances in cutaneous melanoma epidemiology, diagnosis, and treatment, as reported in the journal Cancer. Cancer. 2008; 113:1728-1743.

6. Brenner S and Tamir E. Early detection of melanoma: the best strategy for a favourable prognosis. Clin Dermatol. 2002; 20:203-211.

7. Koomen JM, Smalley KS. Using quantitative proteomic analysis to understand genotype specific intrinsic drug resistance in melanoma. Oncotarget. 2011; 2:329-35.

8. Korotchkina LG, Leontieva OV, Bukreeva EI, Demidenko ZN, Gudkov AV, Blagosklonny MV. The choice between p53-induced senescence and quiescence is determined in part by the mTOR pathway. Aging. 2010; 2:344-52.

9. Haferkamp S, Tran SL, Becker TM, Scurr LL, Kefford RF, Rizos H. The relative contributions of the p53 and pRb pathways in oncogene-induced melanocyte senescence. Aging. 2009; 1:542-56.

10. Bansal R, Nikiforov MA. Pathways of oncogene-induced senescence in human melanocytic cells. Cell Cycle. 2010; 9:2782-8.

11. Huber MA, Beug H, Wirth T. Epithelial-mesenchymal transition: NF-kappaB takes center stage. Cell Cycle. 2004; $3: 1477-80$.

12. Jordan NV, Johnson GL, Abell AN. Tracking the intermediate stages of epithelial-mesenchymal transition in epithelial stem cells and cancer. Cell Cycle. 2011; 10:286573.

13. Tauriello DV, Maurice MM. The various roles of ubiquitin in Wnt pathway regulation. Cell Cycle. 2010; 9:3700-9.

14. Cesi V, Casciati A, Sesti F, Tanno B, Calabretta B, Raschellà G. TGF $\beta$-induced c-Myb affects the expression of EMT-associated genes and promotes invasion of ER+ breast cancer cells. Cell Cycle. 2011; 10:4149-61.

15. Salem AF, Bonuccelli G, Bevilacqua G, Arafat H, Pestell RG, Sotgia F, Lisanti MP. Caveolin-1 promotes pancreatic cancer cell differentiation and restores membranous E-cadherin via suppression of the epithelial-mesenchymal transition. Cell Cycle. 10:3692-700.

16. Gasparotto D, Polesel J, Marzotto A, Colladel R, Piccinin S, Modena P, Grizzo A, Sulfaro S, Serraino D, Barzan L, Doglioni C, Maestro R. Overexpression of TWIST2 correlates with poor prognosis in head and neck squamous cell carcinomas. Oncotarget. 2011; 2:1165-75.

17. Johnson JP. Cell adhesion molecules in the development and progression of malignant melanoma. Cancer Metastasis Rev. 1999; 18:345-357.

18. Maret D, Gruzglin E, Sadr MS, Siu V, Shan W, Koch AW, Seidah NG, Del Maestro RF, Colman DR. Surface expression of precursor $\mathrm{N}$-cadherin promotes tumor cell invasion. Neoplasia. 2010; 12:1066-1080.

19. Rebhun RB, Cheng H, Gershenwald JE, Fan D, Fidler IJ, Langley RR. Constitutive expression of the alpha4 integrin correlates with tumorigenicity and lymph node metastasis of the B16 murine melanoma. Neoplasia. 2010; 12:173-182.

20. Ogita H, Takai Y. Cross-talk among integrin, cadherin, and growth factor receptor: roles of nectin and nectin-like molecule. Int Rev Cytol. 2008; 265:1-54.

21. Ogita H, Takai Y. Nectins and nectin-like molecules: roles in cell adhesion, polarization, movement, and proliferation. IUBMB Life. 2006; 58:334-343.

22. Sato T, Irie K, Ooshio T, Ikeda W, Takai Y. Involvement of heterophilic trans-interaction of NECL-5/Tage4/PVR/ CD155 with nectin-3 information of nectin-and cadherinbased adherens junctions. Genes Cells. 2004; 9:791-799.

23. Mendelsohn CL, Wimmer E and Racaniello VR (). Cellular receptor for poliovirus: molecular cloning, nucleotide sequence, and expression of a new member of the immunoglobulin superfamily. Cell. 1989; 56:855-865.

24. Minami Y, Ikeda W, Kajita M, Fujito T, Amano H, Tamaru Y, Kuramitsu K,Sakamoto Y, Monden M, Takai Y. NECL$5 /$ poliovirus receptor interacts in cis with integrin $\alpha$ vbeta3 and regulates its clustering and focal complex formation. $\mathrm{J}$ Biol Chem. 2007; 282:18481-18496.

25. Kakunaga S, Ikeda W, Shingai T, Fujito A, Yamada Y, Minami T, Imai T, Takai Y. Enhancement of serum and platelet-derived growth factor-induced cell proliferation by NECL-5/Tage4/poliovirus receptor/CD155 through the Ras-Raf-MEK-ERK signaling. J Biol Chem. 2004; 279:36419-36425.

26. Hirota $\mathrm{T}$, Irie $\mathrm{K}$, Okamoto $\mathrm{R}$, Ikeda $\mathrm{W}$, Takai $\mathrm{Y}$. Transcriptional activation of the mouse NECL-5/Tage4/ PVR/CD155 gene by fibroblast growth factor or oncogenic Ras through the Raf-MEK-ERK-AP-1 pathway. Oncogene. 2005; 24:2229-2235.

27. Takai Y, Miyoshi J, Ikeda W, Ogita H. Nectins and nectinlike molecules: roles in contact inhibition of cell movement and proliferation. Nat Rev Mol Cell Biol. 2008; 9:603-615.

28. Fournier G, Garrido-Urbani S, Reymond N, Lopez M. Nectin and nectin-like molecules as markers, actors and targets in cancer. Med Sci. 2010; 26:273-279.

29. Sloan KE, Eustace BK, Stewart JK, Zehetmeier C, Torella C, Simeone M, Roy JE, Unger C, Louis DN, Ilag LL, Jay DG. CD155/PVR plays a key role in cell motility during tumor cell invasion and migration. BMC Cancer. 2004; 4:73-82.

30. Ochiai H, Moore SA, Archer GE, Okamura T, Chewning TA, Marks JR, Sampson JH, Gromeier M. Treatment of intracerebral neoplasia and neoplastic meningitis with regional delivery of oncolytic recombinant poliovirus. Clin Cancer Res. 2004; 10:4831-4838.

31. Merrill MK, Bernhardt G, Sampson JH, Wikstrand CJ, Bigner DD, Gromeier M. Poliovirus receptor CD155targeted oncolysis of glioma. J Neurooncol. 2004; 6:208232.

32. Carlsten M, Norell H, Bryceson YT, Poschke I, Schedvins K, Ljunggren HG, Kiessling R, Malmberg KJ. Primary 
human tumor cells expressing CD155 impair tumor targeting by down-regulating DNAM-1 on NK cells. J Immunol. 2009; 183:4921-4930

33. Suzuki K, Nakamura K, Kato K, Hamada H, Tsukamoto $\mathrm{T}$. Exploration of target molecules for prostate cancer gene therapy. Prostate. 2007; 67:1163-1173

34. Masson D, Jarry A, Baury B, Blanchardie P, Laboisse C, Lustenberger P, Denis MG. Overexpression of the CD155 gene in human colorectal carcinoma. Gut. 2001; 49:236240.

35. Nakai R, Maniwa Y, Tanaka Y, Nishio W, Yoshimura M, Okita Y, Ohbayashi C, Satoh N, Ogita H, Takai Y, Hayashi Y. Overexpression of NECL-5 correlates with unfavorable prognosis in patients with lung adenocarcinoma. Cancer Sci. 2010; 101:1326-1330.

36. Talantov D, Mazumder A, Yu JX, Briggs T, Jiang Y, Backus J, Atkins D, Wang Y. Novel genes associated with malignant melanoma but not benign melanocytic lesions. Clin Cancer Res. 2005; 11:7234-7242.

37. Riker AI, Enkemann SA, Fodstad O, Liu S, Ren S, Morris C, Xi Y, Howell P, Metge B, Samant RS, Shevde LA, Li W, Eschrich S, Daud A, Ju J, Matta J. The gene expression profiles of primary and metastatic melanoma yields a transition point of tumor progression and metastasis. BMC Med Genomics. 2008; 1:1-13.

38. Xu L, Shen SS, Hoshida Y, Subramanian A, Ross K, Brunet JP, Wagner SN, Ramaswamy S, Mesirov JP, Hynes RO. Gene expression changes in an animal melanoma model correlate with aggressiveness of human melanoma metastases. Mol Cancer Res. 2008; 6:760-769.

39. Haqq C, Nosrati M, Sudilovsky D, Crothers J, Khodabakhsh D, Pulliam BL, Federman S, Miller JR 3rd, Allen RE, Singer MI, Leong SP, Ljung BM, Sagebiel RW, KashaniSabet M. The gene expression signatures of melanoma progression. Proc Natl Acad Sci USA. 2005; 102:60926097

40. Cummins DL, Cummins JM, Pantle H, Silverman MA, Leonard AL, Chanmugam A. Cutaneous malignant melanoma. Mayo Clin Proc. 2006; 81:500-507.

41. Crowson AN, Magro CM, Sanchez-Carpintero I. The precursors of malignant melanoma. Recent Results Cancer Res. 2002; 160:75-84.

42. Casado JG, Pawelec G, Morgado S, Sanchez-Correa B, Delgado E, Gayoso I, Duran E, Solana R, Tarazona R. Expression of adhesion molecules and ligands for activating and costimulatory receptors involved in cell-mediated cytotoxicity in a large panel of human melanoma cell lines. Cancer Immunol Immunother. 2009; 58:1517-1526.

43. Pende D, Bottino C, Castriconi R, Cantoni C, Marcenaro S, Rivera P, Spaggiari GM, Dondero A, Carnemolla B, Reymond N, Mingari MC, Lopez M, Moretta L, Moretta A. PVR (CD155) and Nectin-2 (CD112) as ligands of the human DNAM-1 (CD226) activating receptor: involvement in tumor cell lysis. Mol Immunol. 2005. 42:463-469
44. Koike S, Horie H, Ise I, Okitsu A, Yoshida M, Iizuka $\mathrm{N}$, Takeuchi K, Takegami T, Nomoto A The poliovirus receptor protein is produced both as membrane-bound and secreted forms. EMBO J. 1990; 9:3217-3224.

45. Statius Muller MG, van Leeuwen PA, de Lange-De Klerk ES, van Diest PJ, Pijpers R, Ferwerda CC, Vuylsteke RJ, Meijer S. The sentinel lymph node status is an important factor for predicting clinical outcome in patients with Stage I or II cutaneous melanoma. Cancer. 2001; 91:2401-2408.

46. Massi D, Borgognoni L, Franchi A, Martini L, Reali UM, Santucci M. Thick cutaneous malignant melanoma: a reappraisal of prognostic factors. Melanoma Res. 2000; 10:153-164.

47. Danen EH, deVries TJ, Morandini R, Ghanem GG, Ruiter DJ, van Muijen GN. E-cadherin expression in human melanoma. Melanoma Res. 1996; 6:127-131

48. Zheng G, Lyons JG, Tan TK, Wang Y, Hsu TT, Min D, Succar L, Rangan GK, Hu M, Henderson BR, Alexander SI, Harris DC. Disruption of E-cadherin by matrix metalloproteinase directly mediates epithelial-mesenchymal transition downstream of transforming growth factorbeta1 in renal tubular epithelial cells. Am J Pathol. 2009; 175:580-591.

49. Enloe BM, Jay DG. Inhibition of NECL-5 (CD155/PVR) reduces glioblastoma dispersal and decreases MMP-2 expression and activity. J Neurooncol. 2011; 102:225-235.

50. Malaponte G, Zacchia A, Bevelacqua Y, Marconi A, Perrotta R, Mazzarino MC, Cardile V, Stivala F. Coregulated expression of matrix metalloproteinase-2 and transforming growth factor-beta in melanoma development and progression. Oncol Rep. 2010; 24:81-87.

51. Hanahan D, Weinberg RA. The hallmarks of cancer. Cell. 2011; 144:646-674.

52. Castellano G, Torrisi E, Ligresti G, Malaponte G, Militello L, Russo AE, McCubrey JA, Canevari S, Libra M. The involvement of the transcription factor YY1 in cancer development and progression. Cell Cycle. 2009; 8:13671372.

53. Zaravinos A, Spandidos DA. Yin yang 1 expression in human tumors. Cell Cycle. 2010; 9:512-22.

54. Caggia S, Libra M, Malaponte, Cardile V. Modulation of YY1 and p53 expression by transforming growth factor- $\beta 3$ in prostate cell lines. Cytokine. 2011; 56:403-410

55. Castellano G, Torrisi E, Ligresti G, Nicoletti F, Malaponte G, Travali S, McCubrey JA, Canevari S, Libra M. YY1 overexpression in diffuse large B-cell lymphoma is associated with B-cell transformation and tumor progression. Cell Cycle. 2010; 9:557-563.

56. Rothweiler F, Michaelis M, Brauer P, Otte J, Weber K, Fehse B, Doerr HW, Wiese M, Kreuter J, Al-Abed Y, Nicoletti F, Cinatl J Jr. Anticancer effects of the nitric oxide-modified saquinavir derivative saquinavir-NO against multidrug-resistant cancer cells. Neoplasia. 2010; 12:10231030. 